\title{
Research on Making Non-Stationary Random Excitation of Automobile Systems Change Into Stationary One
}

\author{
Wang Fangming, Chai Zhiyong \\ China Automotive Technology \& Research Center, No.3 Bo Xing Liu Lu, Yi Zhuang, Beijing 100176
}

\begin{abstract}
Making non-stationary random excitation in automobile vibration system change into stationary one is that the sample function of random excitation studied is made into a product of two functions, one of which is a definite function, called observation function, the other is stationary random process, called stationary random sample function. This article presented the necessary condition on which the observation function exists and the calculating formula of it, providing a theorems basis for the further research about non-stationary random vibration of automobile systems .
\end{abstract}

Keywords-random vibration, automobile dynamics, non-stationary random excitation

\section{THREE THEOREMS}

Changing non-stationary random excitation change into stationary one is that the sample function of random excitation studied is made into a product of two functions. For one dimension non-stationary excitation $\bar{f}(t)$, we can get:

$$
\bar{f}(t)=g(t) \bar{x}(t)
$$

"_-"denotes random vibration excitation. For multiple dimensions,

$$
\bar{F}(t)=\left\{\begin{array}{c}
\overline{f_{1}}(t) \\
\overline{f_{2}}(t) \\
\cdots \\
\cdots \\
\cdots \\
\overline{f_{n}}(t)
\end{array}\right\}
$$

Can get

$$
\begin{gathered}
\left\{\begin{array}{c}
\overline{f_{1}}(t) \\
\overline{f_{2}}(t) \\
\cdots \\
\cdots \\
\cdots \\
\overline{f_{n}}(t)
\end{array}\right\}=\left[\begin{array}{ccc}
g_{1}(t) & \cdots & 0 \\
\vdots & \ddots & \vdots \\
0 & \cdots & g_{n}(t)
\end{array}\right]\left[\begin{array}{c}
\overline{\bar{x}_{1}}(t) \\
\overline{x_{2}}(t) \\
\cdots \\
\cdots \\
\cdots \\
\overline{x_{n}}(t)
\end{array}\right\} \\
\boldsymbol{G}(\boldsymbol{t})=\left[\begin{array}{ccc}
g_{1}(t) & \cdots & 0 \\
\vdots & \ddots & \vdots \\
0 & \cdots & g_{n}(t)
\end{array}\right\}
\end{gathered}
$$

$$
\bar{X}(t)=\left\{\begin{array}{c}
\overline{x_{1}}(t) \\
\overline{x_{2}}(t) \\
\cdots \\
\cdots \\
\cdots \\
\overline{x_{n}}(t)
\end{array}\right\}
$$

Simply,

$$
\bar{F}(\boldsymbol{t})=\boldsymbol{G}(\boldsymbol{t}) \cdot \bar{X}(\boldsymbol{t})
$$

$g(t)$ or $\boldsymbol{G}(\boldsymbol{t})$ is a definite function or a definite matrix. $g(t)>0$. $\quad \boldsymbol{G}(t)$ is a matrix which can be inversed. $\bar{x}(t)$ or $\overline{\boldsymbol{X}}(\boldsymbol{t})$ is weak stationary random excitation. So the key problem is how the $g(t)$ or $\boldsymbol{G}(\boldsymbol{t})$ is designed.

\section{Theorem 1}

In one dimensional random process, the necessary conditions on which $g(t)$ exist is

$$
\text { 1. } D_{f}(\mathrm{t}) \neq 0, \quad E_{f}(\mathrm{t}) \neq 0
$$

$$
\text { 2. } \sqrt{D_{f}(t)}=\text { C. } E_{f}(\mathrm{t})
$$

$D_{f}(t)$ presents the variance of $\bar{f}(t), E_{f}(t)$ is presents the mean values of $\bar{f}(t)$.

Demonstrations

From

$\bar{f}(t)=g(t) \cdot \bar{x}(t)$

Have

$D_{f}(t)=g(t)^{2} \cdot D_{x}(t), \quad D_{x}(t)$ presents the variance of $\bar{x}(t)$

$E_{f}(t)=g(t) \cdot E_{x}(t), \quad E_{x}(t)$ presents the mean values of

Or

$$
\begin{gathered}
E_{x}(t)=E_{f}(\mathrm{t}) / g(t) \\
D_{x}(t)=D_{f}(\mathrm{t}) / g(t)^{2}
\end{gathered}
$$


A cording to the nature of weak stationary random excitation, we can get

$$
\frac{d}{d_{t}} D_{x}(t)=\frac{g(t)^{2} \dot{D_{f}}(t)-2 g(t) \dot{g}(t) D_{f}(\mathrm{t})}{g(t)^{4}}=0
$$

Thus

$$
g(t)^{2} \dot{D}_{f}(t)-2 g(t) \dot{g}(t) D_{f}(\mathrm{t})=0
$$

In case of $D_{f}(\mathrm{t})>0$,

$$
\begin{gathered}
\dot{g}(t)-\frac{\dot{D_{f}}(t)}{2 D_{f}(\mathrm{t})} g(t)=0 \\
g(t)=C_{1} e^{\int \frac{D_{f}(t)}{2 \dot{D}_{f}(t)} d t}=C_{1} e^{\frac{1}{2} \ln D_{f}(\mathrm{t})} \\
=C_{1} \sqrt{D_{f}(\mathrm{t})}
\end{gathered}
$$

From $E_{x}(t)=E_{f}(\mathrm{t}) / g(t)$

According to the nature of weak stationary random excitation, we obtain

$$
\begin{aligned}
\frac{d}{d_{t}} E_{x}(t) & =\frac{g(t) \dot{E}_{f}(t)-\dot{g}(t) E_{f}(\mathrm{t})}{g(t)^{2}} \\
& =0 \\
g(t) \dot{E}_{f}(t)- & \dot{g}(t) E_{f}(\mathrm{t})=0
\end{aligned}
$$

Here ask for $E_{f}(\mathrm{t}) \neq 0$, then combining eq.(5)

$$
\begin{aligned}
& \quad g(t)=C_{2} e^{\int_{\frac{E_{f}}{E_{f}(t)}} d t}=C_{2} E_{f}(\mathrm{t}) \\
& =C_{1} \sqrt{D_{f}(\mathrm{t})}
\end{aligned}
$$

Thus

$$
\sqrt{D_{f}(\mathrm{t})}=\mathrm{C} \cdot E_{f}(\mathrm{t}), C={ }^{C}{ }_{2} / C_{1}, C \text { is a constant number. }
$$

\section{Theorem 2}

In multiple-dimensional random excitation $\overline{\boldsymbol{F}}(\boldsymbol{t})$, if $D_{f}(t)_{i j} \neq 0,(i \neq j)$, the necessary conditions on which $\boldsymbol{G}(\boldsymbol{t})$ exist is

$$
\begin{gathered}
\text { 1. } D_{F}(t)_{i i} \neq 0, \quad E_{F}(\mathrm{t})_{i} \neq 0 \\
\text { 2. } \alpha \frac{D_{F}(t)_{i j}}{D_{F}(t)_{i j}}+\beta \frac{\dot{D_{F}}(t)_{i i}}{D_{F}(t)_{i i}}+\gamma \frac{D_{F}(t)_{j j}}{D_{F}(t)_{j j}}=0
\end{gathered}
$$

(

$\alpha \beta \gamma$ are constants which are not egual to zero simultaneously )

$$
\text { 3. } \sqrt{D_{F}(t)_{i i}}=C_{i} E_{F}(\mathrm{t})_{i} \quad(i=1,2,3 \cdots \cdots \mathrm{n}),
$$

$\boldsymbol{D}_{\boldsymbol{F}}(\boldsymbol{t})$ is the variance matrix of $\overline{\boldsymbol{F}}(\boldsymbol{t}), \boldsymbol{E}_{\boldsymbol{F}}(\mathbf{t})$ is the mean values of $\overline{\boldsymbol{F}}(\boldsymbol{t})$.

If $D_{F}(t)_{i j}=0(i \neq j)$, only condition 1 and 3 can be considered.

Demonstrations.

From

$$
\bar{F}(\boldsymbol{t})=\boldsymbol{G}(\boldsymbol{t}) \bar{X}(t)
$$

Have

$$
\begin{aligned}
& D_{F}(t)=\boldsymbol{G}(\boldsymbol{t}) \boldsymbol{D}_{\boldsymbol{x}}(\boldsymbol{t}) \boldsymbol{G}(\boldsymbol{t}) \\
& E_{F}(\mathrm{t})=\boldsymbol{G}(\boldsymbol{t}) E_{X}(\mathrm{t})
\end{aligned}
$$

From eq. (7),

$$
D_{X}(t)_{i j}=D_{F}(t)_{i j} / g(t)_{i} g(t)_{j}
$$

Following the nature of stationary random excitation,

$$
\begin{aligned}
& \frac{d}{d_{t}} D_{X}(t) \\
& =\frac{g_{i}(t) g_{j}(t) \dot{D}_{F}(t)_{i j}-D_{F}(t)_{i j} d\left[g_{i}(t) g_{j}(t)\right] / d t}{g_{i}(t)^{2} g_{j}(t)^{2}} \\
& =0
\end{aligned}
$$

Therefore

$g_{i}(t) g_{j}(t) \dot{D}_{F}(t)_{i j}-D_{F}(t)_{i j}\left[\dot{g}_{i}(t) g_{j}(t)+g_{i}(t) \dot{g}_{j}(t)\right]=$

Let $i=j, e q .(9)$ becomes

$$
g_{i}(t)^{2} \dot{D}_{F}(t)_{i i}-2 D_{F}(t)_{i i} g_{i}(t) \dot{g}_{i}(t)=0
$$

Ask for $D_{F}(t)_{i i} \neq 0$, hense

$$
\begin{gathered}
\dot{g}_{i}(t)-\frac{\dot{D}_{F}(t)_{i i}}{2 D_{F}(t)_{i i}} g_{i}(t)=0 \\
g_{i}(t)=C_{i} e^{\int \frac{\dot{D}_{F}(t)_{i i}}{2 D_{F}(t)_{i i}} d t} \\
=C_{i} \sqrt{D_{F}(t)_{i i}}
\end{gathered}
$$

$C_{i}$ is constant

$$
(i=1,2,3 \cdots \cdots n)
$$

In case of $D_{F}(t)_{i j} \neq 0, \quad$ take eq.(11) into eq.(9) and get

$$
\begin{gathered}
C_{i} C_{j} \dot{D}_{F}(t)_{i j} \sqrt{D_{F}(t)_{i i} D_{F}(t)_{j j}}- \\
D_{F}(t)_{i j}\left[C_{i} C_{j} \sqrt{\frac{D_{F}(t)_{j j}}{D_{F}(t)_{i i}}} \frac{\dot{D}_{F}(t)_{i i}}{2}+C_{i} C_{j} \sqrt{\frac{D_{F}(t)_{i i}}{D_{F}(t)_{j j}}} \frac{\dot{D}_{F}(t)_{j j}}{2}\right]=0
\end{gathered}
$$

Since $\quad C_{i}$ and $C_{j}$ are arbitrary constants, through simplifying, eq.(12) can be written as

$$
\alpha \frac{\dot{D_{F}}(t)_{i j}}{D_{F}(t)_{i j}}+\beta \frac{\dot{D_{F}}(t)_{i i}}{D_{F}(t)_{i i}}+\gamma \frac{\dot{D_{F}}(t)_{j j}}{D_{F}(t)_{j j}}=0
$$


$\alpha \beta \gamma$ are constants which are not egual to zero simultaneously

From $\boldsymbol{E}_{\boldsymbol{F}}(\mathbf{t})=\boldsymbol{G}(\boldsymbol{t}) \boldsymbol{E}_{\boldsymbol{X}}(\mathbf{t})$, have

$$
\begin{gathered}
E_{F}(t)_{i}=g_{i}(t) E_{X}(t)_{i} \\
E_{X}(t)_{i}=\frac{E_{F}(t)_{i}}{g_{i}(t)} \quad g_{i}(t) \neq 0,(i=1,2,3 \cdots \cdots n)
\end{gathered}
$$

According to the nature of stationary random excitation, get

$$
\frac{d}{d t} E_{X}(t)_{i}=0
$$

Taking eq.(11) into account, by the same way as we demonstrate Theorem 1, we have

$$
\begin{gathered}
\sqrt{D_{F}(t)_{i i}}=C_{i} E_{F}(\mathrm{t})_{i} \\
\begin{array}{c}
D_{F}(t)_{i i} \neq 0, \quad E_{F}(\mathrm{t})_{i} \neq 0 \quad(i=1,2,3 \\
\cdots \cdots n)
\end{array}
\end{gathered}
$$

If $D_{F}(t)_{i j}=0(i \neq \mathrm{j})$, only eq.(14) is necessary.

Theorem 3 In case Theorem 1 or Theorem 2 is met, definite function $g(t)$ or matrix $\boldsymbol{G}(\boldsymbol{t})$ can be calculated as below

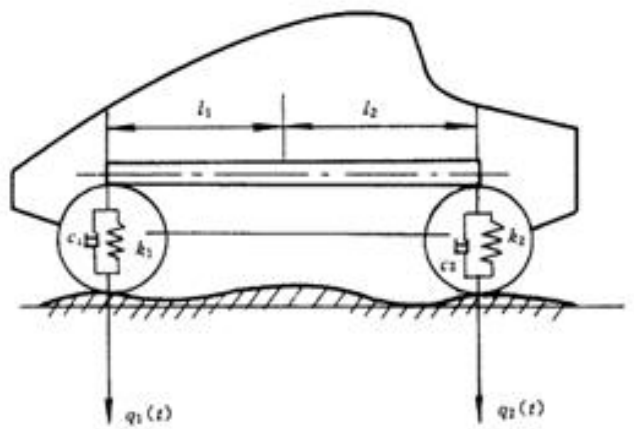

(a)

Simply, vibration model of vehicle can described as (ref.1)

$$
M \ddot{\bar{Z}}+C \dot{\bar{Z}}+K \bar{Z}=\bar{F}
$$

If $\overline{\boldsymbol{F}}=\left\{\begin{array}{l}\bar{f}_{1}(t) \\ \bar{f}_{2}(t)\end{array}\right\} \quad \bar{A}$ and $\bar{B}$ are not relative random number

Have

$D_{F}=\left[\begin{array}{cc}\boldsymbol{D}_{\boldsymbol{A}} \sin ^{2} \omega_{1} t & \mathbf{0} \\ \mathbf{0} & \boldsymbol{D}_{\boldsymbol{B}} \sin ^{2} \omega_{2} t\end{array}\right]$

So $D_{\ddot{Z}}$ and $D_{\dot{Z}}$ or $D_{Z}$ can be calculated easily in (15), which are important for the vehicle design.

$$
\begin{aligned}
& \text { 1. } g(t)=C \sqrt{D_{f}(t)} \text { or } g(t)=C E_{f}(t) \\
& \text { 2. } g_{i}(t)=C_{i} \sqrt{D_{F}(t)_{i i}} \text { or } g_{i}(t)=C_{i} E_{F}(\mathrm{t})_{i}
\end{aligned}
$$

$C$ and $C_{i}$ are arbitrary constants

When we demonstrate Theorem 1 or Theorem 2, Theorem 3 is proved simultaneously.

Example

Take a two dimensional random excitation

$$
\left\{\begin{array}{l}
\bar{f}_{1}(t) \\
\bar{f}_{2}(t)
\end{array}\right\}=\left\{\begin{array}{l}
\bar{A} \sin \omega_{1} t \\
\bar{B} \sin \omega_{2} t
\end{array}\right\}
$$

If $\omega_{1} t \neq 0, \omega_{2} t \neq 0$ this random excitation is proved to meet the Theorem 1, thus

$$
\begin{aligned}
& g_{1}(t)=C_{1} \sqrt{D_{F}(t)_{11}}=C_{1} \sqrt{D_{A}} \sin \omega_{1} t \\
& g_{2}(t)=C_{2} \sqrt{D_{F}(t)_{22}}=C_{2} \sqrt{D_{B}} \sin \omega_{2} t
\end{aligned}
$$

$D_{A}$ or $D_{B}$ is the variance of $\bar{A}$ or $\bar{B}$

Fig. (a) and Fig. (b) are vibration model of vehicle.

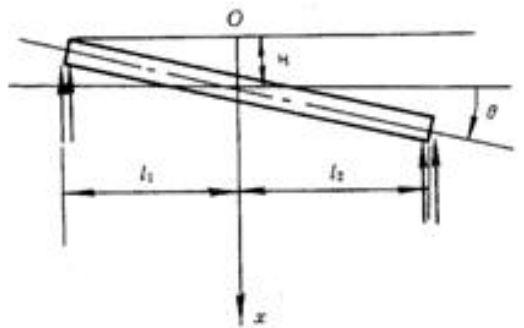

(b)

\section{RESULT AND DISCUSION}

Now we see, the condition about making multi-dimensional non-stationary random process change stationary one is more harshly than that about making one dimensional non-stationary random process change stationary one. One dimensional non-stationary random process is a special case of the multi-dimensional non-stationary random process. The results provided in this article are the same with those derived from ref.2

\section{REFERENCE}

[1] Zhuang Biaozhong, Non-linear Random Vibration Theory and Its Application, Zhejiang University Press, China, 1986

[2] Wang Fangming, Shen Zhengbin, Journal of Vibration and Shock (Chinese), Vol.8, No.3 (1989), 55. 